\title{
Impacto del adalimumab sobre el infiltrado de linfocitos T en pacientes con psoriasis
}

Impact of Adalimumab on lymphocyte T infiltration in patiensts with psoriasis.

\section{Ismael Cheng-Flores' ${ }^{\mathbf{1}}$, Mario Amaya-Guerra', Diana González-Cabello}

1. Servicio de Dermatología, Hospital de especialidades $N^{0} 25$, Centro Médico Nacional del Noreste, Instituto Mexicano del Seguro Social, Monterrey, México.

2. Servicio de Anatomía Patológica, Unidad médica de alta especialidad $\mathrm{N}^{\circ} 34$, Centro Médico Nacional del Noreste, Instituto Mexicano del Seguro Social, Monterrey, México.

\section{RESUMEN}

OBJETIVO. Demostrar el impacto del adalimumab en la reducción del infiltrado de linfocitos T en la piel.

MATERIAL Y MÉTODOS. Es un estudio experimental de antes y después, que se realizó de marzo a diciembre del 2011. Se incluyeron todos los pacientes mayores de 18 años con psoriasis y un PASI mayor de 10, que iniciaron tratamiento con adalimumab. Los criterios de exclusión fueron: pacientes que hubieran recibido tratamiento previo con infliximab, etarnecept, ciclosporina o metotrexato y pacientes que no hubieran suspendido algún tratamiento tópico, por lo menos, dos meses antes del estudio. Se les tomó biopsia de piel antes del inicio del tratamiento y a la semana 28, las cuales se analizaron con técnicas de hematoxilina y eosina, e inmunohistoquímica. Se contó el número linfocitos por medio del microscopio óptico a 100X. Para el análisis estadístico, se utilizaron las pruebas t de Student y ji al cuadrado, con el paquete estadístico SPSS $15{ }^{\circledR}$.

RESULTADOS. Se incluyeron 22 pacientes con psoriasis. La mediana de la edad del

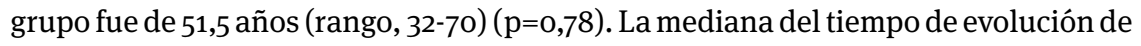

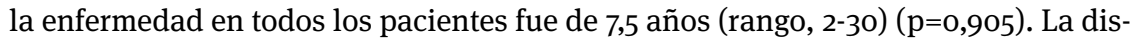
minución de los linfocitos T CD8+ después del tratamiento fue del 58,8 \% ( $\mathrm{p}=0,000)$ y de los linfocitos T CD4+ fue del 41,5\% (p=o,ooo) después del tratamiento.

CONCLUSIONES: El tratamiento con adalimumab disminuye significativamente el infiltrado linfocitico en la epidermis.

PALABRAS CLAVE: adalimumab, linfocitos CD4, linfocitos CD8.

\section{SUMMARY}

OBJETIVE: To evaluate the impact of adalimumab in reducing T lymphocyte infiltration in the skin of patients with psoriasis.

MATERIAL AND METHODS: A pre-test - post-test study was conducted from March until December 2011. Patients with psoriasis older than 18 years of age with a PASI $>10$ were included. Patients who had received treatment with infliximab, etanercept, cyclosporine, metotrexate and those not stopping any topical treatment for at least two months before enrolled were excluded. A skin biopsy was taken before and at 28 weeks of treatment with adalimumab. Biopsies were examined by $\mathrm{H}$ and $\mathrm{E}$ and
Correspondencia:

Ismael Cheng-Flores

Email:

imederm@outlook.com

Recibido: 2 de marzo de 2015 Aceptado: 24 de noviembre 2015

No se reportan conflictos de interés. 
inmunohistochemistry tecniques. T Student and chi square tests were used for the statistical analysis.

RESULTS: Twenty two patients with psoriasis were included. The mean age was 51.6 years (range, 32-70) $(\mathrm{p}=0.70)$. The time of progression of the disease was 7.5 years (range, 2-30) ( $\mathrm{p}=0.905)$. The CD4+ T lymphocyte counts was 58.8\% ( $\mathrm{p}=0.000)$ lower after treatment. The CD8+ T lymphocyte counts was $41.5 \%(\mathrm{p}=0.000)$ lower after treatment.

CONCLUSIONS: Adalimumab reduces significantly the Tlymphocytes infiltration in the epidermis of patients with psoriasis.

KEYWORDS: Adalimumab, CD4 lymphocytes, CD8 lymphocytes.

\section{INTRODUCCIÓN}

La psoriasis es una enfermedad sistémica inflamatoria crónica, con predominio cutáneo ${ }^{1}$. Hay varios estudios que sugieren que se trata de una enfermedad autoinmunitaria, mediada sobre todo por linfocitos T. Estas células son las principales efectoras de la patogenia de la enfermedad. A pesar de múltiples investigaciones, aún no se ha podido encontrar cuáles son los antígenos responsables de desencadenar la reacción inmunitaria. El linfocito T es la célula que predomina en el infiltrado de la lesión psoriásica. Tanto el linfocito T CD4+ como el linfocito T CD8+, se encuentran en las lesiones de psoriasis. En la placa de psoriasis inicialmente llegan los linfocitos T CD4+ activados a la epidermis y, cuando hay una resolución de la enfermedad, se observa predominio de linfocitos T CD 8+ y disminución de linfocitos T CD $4+^{2,3}$. En la piel enferma, los linfocitos $\mathrm{T}$ activados segregan una serie de citocinas que pueden ser causantes de muchas de las características de esta enfermedad inflamatoria ${ }^{4,5}$. El factor de necrosis tumoral alfa (TNF- $\alpha$ ), la IL-3, la IL-6, el GM-CSF y el IFN-g, son responsables de la proliferación de la epidermis. El TNF- $\alpha$ incrementa los niveles de antileucoproteinasa y $\beta$ defensinas.

El adalimumab es el primer anticuerpo monoclonal anti-TNF- $\alpha$ completamente humano. Se une al TNF- $\alpha$ soluble y a las membranas, bloqueando su interacción con los receptores celulares del $\mathrm{TNF}^{6-8}$. La dosis es de 80 mg por vía subcutánea en la semana o, seguidos de 40 mg cada dos semanas desde la semana 1 en adelante. Los mejores resultados se obtienen en esquemas continuos, observándose disminución de la eficacia al suspender y reiniciar el tratamiento.

El objetivo del estudio fue demostrar el impacto del adalimumab en el infiltrado de los linfocitos T evaluado por inmunohistoquímica en pacientes con psoriasis.

\section{MATERIALES Y MÉTODOS}

Se trata de un estudio experimental de antes y después. Se llevó a cabo de marzo del 2011 a diciembre del 2011.

Los criterios de inclusión fueron: pacientes con psoriasis, mayores de 18 años con un índice de gravedad de psoriasis (PASI) mayor de 10. Los criterios de exclusión fueron: todos los pacientes con tratamiento previo con infliximab, etanercept, ciclosporina o metotrexato y aquellos que no hubieran suspendido algún tratamiento tópico, por lo menos, dos meses antes del estudio. Para el tamaño de la muestra se incluyeron todos los pacientes con psoriasis grave a quienes se les hubiera iniciado tratamiento con adalimumab. Se realizó la técnica de muestreo por conveniencia.

A los pacientes se les tomó biopsia de piel antes del inicio del tratamiento y a la semana 28 , las cuales se analizaron con las técnicas de hematoxilina y eosina e inmunohistoquímica para linfocitos T CD8+ y linfocitos T CD4+; se contó el número de linfocitos por medio del microscopio óptico a $100 \mathrm{X}$.

Para la estadística descriptiva, se utilizaron medidas de tendencia central y de dispersión, así como frecuencias simples y porcentajes. Para el análisis inferencial, se utilizaron las pruebas de t de Student y de ji al cuadrado, con el paquete estadístico SPSS ${ }^{\circledR}$, versión 15.

Este protocolo de estudio se realizó de acuerdo con los reglamentos éticos del hospital y con la autorización del Comité de Ética, siguiendo el reglamento de la Ley General de Salud de la Secretaría de Salud y Asistencia del 2011, en materia de investigación en seres humanos y, por último, se tomó en cuenta la Declaración Internacional de Helsinki de 1975, modificada en Seúl (Corea) en 2008.

\section{RESULTADOS}

Se incluyeron 22 pacientes con psoriasis; siete eran mu- 


\begin{tabular}{cccc}
\hline \hline & MUJERES & HOMBRES & P \\
Sexo & 7 & 15 & 0,088 \\
Edad (años) & $47,5(32-67)$ & $53(30-72)$ & 0,78 \\
$\begin{array}{c}\text { Evolución de } \\
\text { la enfermedad (años) }\end{array}$ & $4(2-5)$ & $16(6-30)$ & 0,905 \\
\hline \hline
\end{tabular}

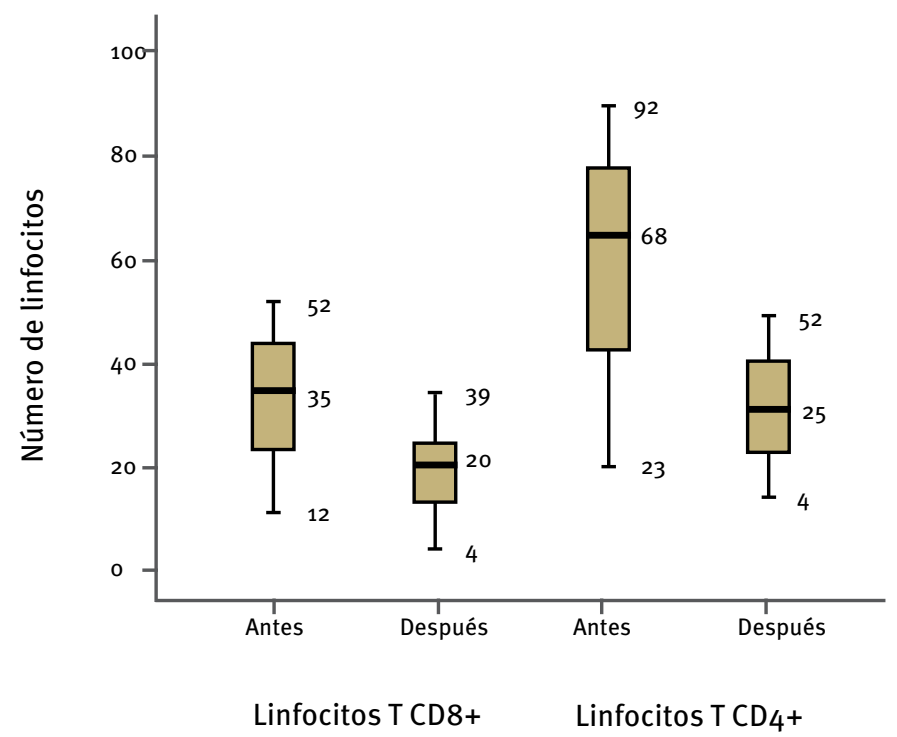

TABLA 1. Características generales de los 22 pacientes con psoriasis en tratamiento con adalimumab. jeres (31,8 \%) y quince eran hombres $(68,2 \%)(p=0,088)$. La mediana de la edad del grupo fue de 51,5 años (rango, 32-70); la edad de las mujeres fue de 47,5 años (rango, $32-67$ ) y la de los hombres fue de 53 años (rango, 30-72) $(p=0,78)$. La mediana del tiempo de evolución de la enfermedad en todos los pacientes fue de 7,5 años (rango, 2-30), el tiempo de evolución de la enfermedad en las mujeres fue de 4 años (rango, 2-5), el tiempo de evolución de la enfermedad en hombres fue de 16 años (rango, 6-30) $(\mathrm{p}=0,905)$ (TABLA 1).

La mediana basal del número de linfocitos T $\mathrm{CD}^{+}$fue de 35 (rango, 12-52) y a la semana 28 del inicio del tratamiento con adalimumab fue de 20,5 (rango, 4-39). Esto concuerda con una disminución del 58,8 \% después del tratamiento $(\mathrm{p}=0,000)$. La mediana basal del número de linfocitos T CD4+ fue de 68 (rango, 23-92) y a la semana 28 del inicio del tratamiento con adalimumab fue de 25 (rango, 4-52). Esto concuerda con una disminución de $41,5 \%$ después del tratamiento $(\mathrm{p}=0,000)$ (FIGURA 1).

\section{DISCUSIÓN}

El adalimumab es un anticuerpo monoclonal que se fija al
TNF-a, el cual participa del desarrollo y el mantenimiento de la psoriasis, incluyendo la proliferación de queratinocitos y el reclutamiento de linfocitos T en la piel ${ }^{9}$.

Los resultados finales de este estudio demuestran que hay un predominio de linfocitos T CD4+ en la epidermis. Los primeros estudios del infiltrado linfocítico en la piel de los pacientes con psoriasis los realizó Morganroth en 1991, cuando utilizaron por primera vez técnicas de inmunohistoquímica para diferenciar linfocitos $\mathrm{T}$ CD4+ y linfocitos T CD8+; se evidenció que los linfocitos T CD4+ eran el 36,6 $\pm 3,1 \%$ y los linfocitos T CD8+

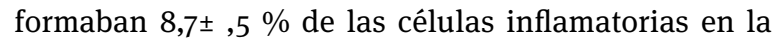
epidermis y en la dermis; concluyeron que los linfocitos T CD8+ predominaban en la epidermis y los linfocitos T CD4+ en la dermis, pero, a diferencia del estudio de Morganroth, en este estudio sólo se contó el número de linfocitos T en la epidermis y ellos contaron el infiltrado en la epidermis y en la dermis ${ }^{9}$. Los resultados finales encontrados en este estudio evalúan la efectividad del adalimumab sobre la disminución del infiltrado linfocítico. No hay estudios previos que midan la reacción después del tratamiento con adalimumab.

Hay pocos estudios en los que se evalúen los trata- 
mientos biológicos a nivel histopatológico con técnicas de inmunohistoquímica. Gómez-Mateo, et al., evaluaron los cambios histopatológicos y la expresión de los marcadores de proliferación y apoptosis en biopsias cutáneas secuenciales de pacientes con psoriasis tratados con infliximab. Al final del estudio, observaron la desaparición de las características histológicas de la psoriasis y disminución de la proliferación epidérmica, pero no encontraron que el tratamiento con infliximab produjera apoptosis en queratinocitos ni producción de anticuerpos anticaspasas ${ }^{10}$.

En otro estudio de Bovenchen, se demostró que el tratamiento con alefacept producía una reducción del infiltrado de linfocitos T CD2+, CD 25+, CD 45 RO+, CD 8+ y $\mathrm{CD}_{4}+$ en las placas de los pacientes con psoriasis ${ }^{11}$.

Aunque se sabe que hay un aumento en el número de linfocitos $\mathrm{T}$ en la piel de pacientes con psoriasis, en algunos informes de casos se ha reportado la disminución de estos linfocitos en pacientes con linfopenia idiopática; estos pacientes presentan una predisposición a infecciones oportunistas y un mayor riesgo de cáncer. Los reportes de casos informan que los pacientes con dicha enfermedad presentan cuadros graves de psoriasis. Esto se debe a una alteración en la regulación de reclutamiento de los linfocitos T CD4+ y linfocitos T FOX $\mathrm{P}_{3}+{ }^{12}$.

En los estudios recientes se sugiere la importancia de la regulación epigenética en el desarrollo de la psoriasis. Park, et al., por medio de la técnica de secuenciación de la metilación del ADN, demostraron que los linfocitos $\mathrm{T} \mathrm{CD}_{4}{ }^{+}$en pacientes con psoriasis tienen niveles globales de metilación más altos que los linfocitos $\mathrm{T}$ CD4+ en pacientes sanos ${ }^{13}$.

Posteriormente, Zhang encontró que esta metilación aberrante se encontraba en los dominios genéticos relacionados con la reacción inmunitaria y la apoptosis. Por tanto, una hipermetilación anormal podría ser responsable de que algunos genes involucrados en el desarrollo de la psoriasis tengan una expresión defectuosa ${ }^{14}$.

\section{CONCLUSIONES}

La psoriasis en una enfermedad sistémica con muchas opciones terapéuticas; la mayoría de las veces, algunos tratamientos están asociados con efectos adversos o escasa reacción terapéutica. El tratamiento para la psoriasis con adalimumab se considera ideal en pacientes seleccionados. Hasta la fecha son pocos los estudios en los cuales se evalúa la eficacia del tratamiento a nivel histológico. En este estudio, el tratamiento con adalimumab disminuyó el infiltrado linfocítico en la epidermis de pacientes con psoriasis. Sin embargo, se requieren más estudios, sobre todo ensayos clínicos controlados con un mayor número de pacientes, para tener mayor validez estadística.

\section{REFERENCIAS}

1. Boehncke WH, Sterry W. Psoriasis - a systemic inflammatory disorder: clinic, pathogenesis and therapeutic perspectives. J Dtsch Dermatol Ges. 2009;7:946-52.

2. Harvell JD, Nowfar-Rad M, Sundram U. An immunohistochemical study of CD, CD8, TIA- 1 and CD56 subsets in inflammatory skin disease. J Cutan Pathol. 2003;30:108-13.

3. Dikicioglu E, Ergin F, Unal E. Nature of cell kinetics in psoriatic epidermis. J Cut Pathol. 2007;34:257-63.

4. Prinzt JC. Role of $\mathrm{T}$ cell in psoriasis. J Eur Acad Dermatol Venereol. 2003;17:257-70.

5. Smith $\mathrm{CH}$, Baker JNWN. Cell trafficking and role of adhesion molecules in psoriasis. In: van de Kerkkhof PCM, Bos JD, editors. Pathogenetic aspects of psoriasis. New York: Elsevier; 1995. p. 151-60.

6. Romero WA, De la Cruz C. Recomendaciones para uso de agentes biológicos en psoriasis. Revista Chilena de Dermatología. 2010;26:55-71.

7. Menter A, Tyrin SK, Kimball AB, Leonardi CL, Langley RG, Strober BE. Adalimumab therapy for moderate to severe psoriasis: A randomized, controlled phase III trial. J Am Acad Dermatol. 2008;58:598-606.

8. Saurat JH, Stingl G, Dubertret L, Papp K, Langley RG, OtorneJPl, CHAMPIONS Study Investigators. Efficacy and safety results from the randomized controlled comparative study of adalimumab vs.metotrexate vs. placebo in patients whith psoriasis (CHAMPION). Br J Dermatol. 2008;158:558-66.

9. Morganroth GS, Chan LS, Weintein GD, Voorhes JJ, Cooper KD. Proliferating cells in psoriatic dermis are comprised primarily of T cells, endothelial cells, and factor XIIIa+ perivascular dendritic cells. J Invest Dermatol. 1991;96:333-40.

10. Gómez-Mateo C, Ávalos-Peralta SP, Ríos-Martín JJ, CarrizosaEsquivel AM, González-Cámpora R, Camacho-Martínez F. Evaluación histológica secuencial de marcadores de proliferación y apoptosis durante el tratamiento de la psoriasis con anti-factor de necrosis tumoral alfa (infliximab). Actas Dermosifiliogr. 2009;100:420-4.

11. Bovenschen HJ, Gerritsen WJ, van Rens DW, Seyger MM. Explorative inmunohistochemical study to evaluate the addition of topical corticosteroids in the early phase of alefacept treatment for psoriasis. Arch Dermatol Res. 2007;298:457-63.

12. Bauroudijian B, Viguier M, Battistela M, Beneton N, Pages C. Psoriasis associated with idiopathic $\mathrm{CD}_{4}+\mathrm{T}$-cell lymphopenia: A regulatory T-cell defect? Br J Dermatol. 2014;171:186-9.

13. Park GT, Han J, Park SG, Kim TY. DNA methylation analysys of $\mathrm{CD}_{4}+\mathrm{T}$ cell in patients with psoriasis. Arc Dermatol Res. 2014;306:259-68.

14. Zhang P, Zhao M, Liangh G, Yin G, Huang D. Whole-genome DNA methylation in skin lesions from patients with psoriasis vulgar. J Autoinmmun.2013;41:17-24. 